\title{
Parisian Options - The Implied Barrier Concept
}

\author{
Jasper Anderluh and Hans van der Weide \\ Delft Institute of Applied Mathematics, Delft University of Technology \\ Mekelweg 4, 2628 CD, Delft, The Netherlands \\ j.h.m.anderluh@ewi.tudelft.nl
}

\begin{abstract}
Research into the direction of specific exotic options - like the Parisians - is often driven by the analysis of structured products. These products contain features that are similar to exotic options. Exchangetrading of the pure exotics is very rare. In the period of rising stock markets, investors were less interested in buying bonds. In order to regain their interest, firms added extra features to the bonds they wanted to issue. One of these features is the right of the bond holder to convert the bond into a given number of stocks under certain conditions. Bonds with this feature are called convertible bonds and are nowadays very common. Most convertible bonds can be re-called by the issuer when the convertible trades above some level for some period. Modelling this feature corresponds to valuation of a Parisian option. In this paper we will point out how we quickly can approximate the Parisian option price by using a standard barrier option with a modified barrier. This is common practice for traders; they increase or decrease the barrier a bit. Here we want to argue what that bit should be. First we will introduce the Parisian contract. Thereafter we list the methods of valuing the Parisian, followed by a section about the implied barrier method. Here we will use concepts from the theory on Brownian excursions and exploit them to derive prices for Parisians that are already in the excursion. We will conclude with a numerical example.
\end{abstract}

\section{The Parisian Contract}

Let $\left\{S_{t}, \mathcal{F}_{t} ; t \geq 0\right\}$ be a process defined on the filtered probability space $\left(\Omega, \mathcal{F}, \mathcal{F}_{t}\right.$, $\mathbb{P})$. According to the Black-Scholes model we have for the risk neutral price process

$$
S_{t}=s_{0} \exp \left(\left(r-0.5 \sigma^{2}\right) t+\sigma B_{t}\right),
$$

where $\left\{B_{t}, \mathcal{F}_{t} ; t \geq 0\right\}$ denotes a standard Brownian Motion, $s_{0}$ the initial value of the stock, $r$ the interest rate and $\sigma$ the volatility. We can use this risk-neutral stock price process to calculate the price of a derivative $V_{\Phi}$ with some (path dependent) pay-off $\Phi\left(\left(S_{t}\right)_{0 \leq t \leq T}\right)$ at time $T$ by,

$$
V_{\Phi}=e^{-r T} \mathbb{E}\left[\Phi\left(\left(S_{t}\right)_{0 \leq t \leq T}\right)\right] .
$$

Here $\Phi$ is the contract function. A standard barrier option is a derivative that pays off like a put or a call that knocks in or out as soon as the stock price hits 
some level. The Parisian option is like a barrier, but we do not only assume the stock price to hit some level, but also to stay above or below that level for a given time. For a given $t$ let $\left(\gamma_{t}, \beta_{t}\right)$ be the excursion from level $L$ straddling $t$, i.e.

$$
\gamma_{t}:=\sup _{s \leq t}\left\{S_{s}=L\right\} \quad \text { and } \quad \beta_{t}:=\inf _{s \geq t}\left\{S_{s}=L\right\}
$$

Now we can use these random variables to define $T_{D}^{-}$, the first time $\left(S_{t}\right)_{t \geq 0}$ is below $L$ longer than $D$ and $T_{D}^{+}$the equivalent random time for staying above $L$ by

$$
T_{D}^{-}:=\inf _{t>0}\left\{t-\gamma_{t}>D ; S_{t}<L\right\} \quad \text { and } \quad T_{D}^{+}:=\inf _{t>0}\left\{t-\gamma_{t}>D ; S_{t}>L\right\}
$$

For an up and down Parisian we use $T_{D}^{+}$respectively $T_{D}^{-}$. If it is a knock in we consider the set $\left\{T_{D}^{ \pm} \leq T\right\}$ otherwise we consider its complement. With this notation we can write pricing formulae for all kinds of Parisian options, e.g. the Parisian down and in call is given by

$$
V_{P D I C}=e^{-r T} \mathbb{E}\left[\left(S_{T}-X\right)^{+} 1_{\left\{T_{D}^{-} \leq T\right\}}\right]
$$

where $X$ denotes the strike of the call. By changing the set of the indicator, we can calculate all variations of down/out and up/in. By changing the left part of the pay-off, we can compute prices for Parisian puts instead of calls.

\section{Valuation Methods for Parisians}

There are two well-known methods for valuing Parisians. One method [6] uses the Black-Scholes PDE with boundary conditions adapted to the Parisian contract. This modified PDE is then solved by numerical methods. The advantage of this method is, that the pricing framework is flexible, i.e. it is possible to deal with discrete dividends and early exercise. The disadvantage is that the convergence is slow. There are methods using some pre-calculated values [5] to increase this convergence. This method is relatively time-consuming in the sense that it is inappropriate for real-time option pricing

Another method calculates the Laplace Transforms of these option prices [2]. There exist quick inversion algorithms [1] to obtain the prices within a given error bound.

Here we propose another method, the so-called implied barrier method. This method consists of coming up with an implied barrier $L^{*}$. Then the standard barrier option with barrier $L^{*}$ and the remaining parameters equal to the Parisian has the same price as the Parisian. This method only uses standard barrier formulae for the European case and obtaining prices is neither difficult nor timeconsuming. 


\section{The Implied Barrier}

Explaining the implied barrier method is threefold. At first we define the implied barrier and give a numerical example. Then we come up with heuristics about what the implied barrier should be and use excursion theory to obtain a formula for it. Finally, we will show for a practical example that prices are quite close. Denoting the first time $\left(S_{t}\right)$ hits $L$ by $T_{L}$, the price of a call with barrier $L^{*}$ is given by

$$
V_{D I C}:=e^{-r T} \mathbb{E}\left[\left(S_{T}-X\right)^{+} 1_{\left\{T_{L^{*}} \leq T\right\}}\right] .
$$

We know that for $L=L^{*}$ the Parisian option should have a value smaller than this standard barrier, but positive. By decreasing $L^{*}$ we can have the value of this barrier every value between the $\left(L^{*}=L\right)$-case and 0 , so for some particular $L^{*}$ the standard barrier has the same price as the Parisian. This particular value of $L^{*}$ is defined as the implied barrier and can formally be stated as:

\section{Definition}

Let $\Phi\left(S_{T}\right)$ the vanilla part of the payoff of a Parisian Up contract. Then for $L>S_{0}$ the implied barrier $L^{*}$ is defined by

$$
\mathbb{E}\left[\Phi\left(S_{T}\right) 1_{\left\{T_{D, L}^{+} \leq T\right\}}\right]=\mathbb{E}\left[\Phi\left(S_{T}\right) 1_{\left\{T_{L^{*}} \leq T\right\}}\right] .
$$

For $L<S_{0}$ we define the implied barrier $L^{*}$ by

$$
\mathbb{E}\left[\Phi(S T) 1_{\left\{T_{D, L}^{-} \leq T\right\}}\right]=\mathbb{E}\left[\Phi\left(S_{T}\right) 1_{\left\{T_{\left.L^{*} \leq T\right\}}\right] .}\right] .
$$

By continuity it is clear that $L^{*}$ always exists. In the graph in figure 1 we show the implied barrier for a down-and-in Parisian call for different times to maturity and different values of $s_{0}$. The Parisian prices we needed for finding this barrier are computed by a numerical inversion of the Laplace Transforms. For this particular option the graph shows that the implied barrier is in the same range for different $T$ and $s_{0}$. However, by basic monotonicity arguments we have the following proposition.

\section{Proposition}

Let $\Phi\left(S_{T}\right)$ be the vanilla part of a Parisian contract. Suppose for a down contract with $L<S_{0}$ that for some finite $T>D>0$ we have

$$
\mathbb{E}\left[\Phi\left(S_{T}\right) 1_{\left\{T_{D, L}^{-} \leq T\right\}}\right]>0 .
$$

Then $L^{*}(t)$, the implied barrier for this contract with maturity $t$ cannot be the same for every $t \in[D, T]$. Moreover $L^{*}\left(s_{0}\right)$, the implied barrier for this contract with initial stock price $s_{0}$ cannot be the same for every $s_{0}>L$.

Considering the scale of the vertical axis in the figure on the next page, we will try to approximate this implied barrier by a constant. In the next section we arrive intuitively at the approximation. Using this approximation we come up with a numerical example. 


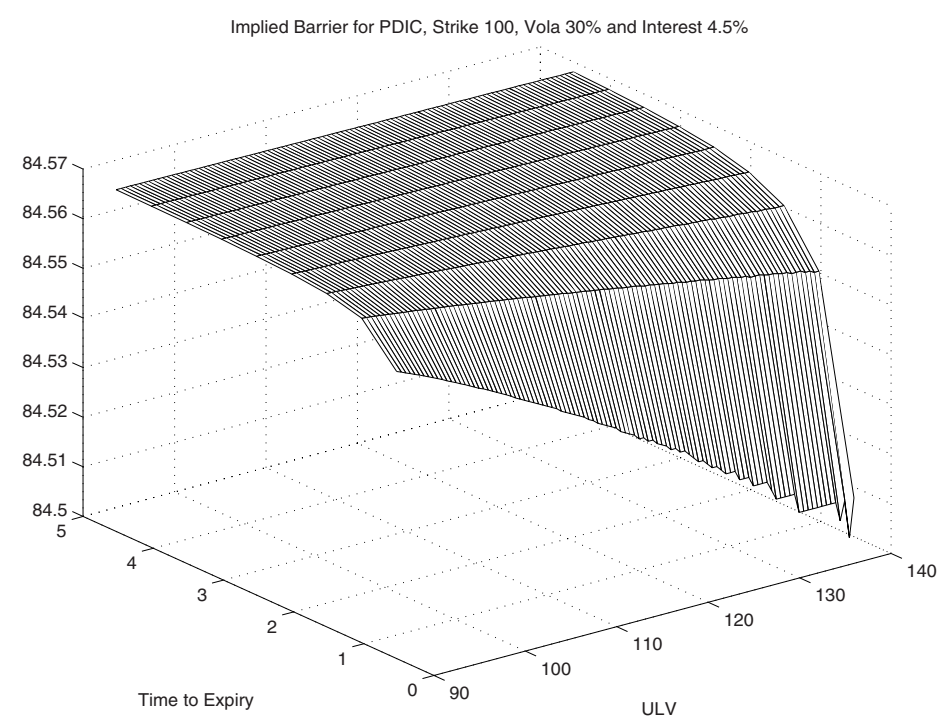

Fig. 1. Implied Barrier vs $s_{0}$ and $T$

\section{Approximating the Implied Barrier}

In order to arrive at the approximation, we argue that the implied barrier should be - whether the contract is of the up or down type - the minimum or maximum we expect $\left(S_{t}\right)$ to attain, given that the stock follows a path in the Parisian set. For the Parisian contracts we are interested in the set $\left\{T_{D}^{ \pm} \leq T\right\}$ and its complement. So, either the realizations of $\left(S_{t}\right)$ do contain an excursion in the right direction w.r.t. to $L$ longer than $D$ or not. If we consider for example the down and in version and divide the stock path into its excursions from $L$, we can compute the expected minimum that is attained in each separate excursion below $L$. The minimum the stock path attains up to time $T$ is the minimum of all these expected minima per excursion. We know from excursion theory that the expected minimum of an excursion decreases when the length of the excursion increases. In this down and in case we know that we need to have at least one excursion longer than $D$, so the expected minimum of the knocking-in stock paths should be below the minimum that we expect to attain during an excursion below $L$ of length $D$. Using Brownian excursion theory [3,4] we have for the expected minimum $m_{D}$ of an Brownian excursion with length $D$

$$
\mathbb{E}\left[m_{D}\right]=\sqrt{\frac{D \pi}{2}} .
$$

As we are dealing with geometric Brownian motion, this term will appear within an exponential. Furthermore we are considering an excursion around the level $L$, so we find the following approximation $\tilde{L}^{*}$ for the implied barrier $L^{*}$ 


$$
\tilde{L}^{*}:=L \exp \left(-\sigma \sqrt{D} e^{-\frac{m^{2}}{2} D} \sqrt{\frac{\pi}{2}}\right) \quad \text { where } m:=\frac{r-0.5 \sigma^{2}}{\sigma} .
$$

Here the $m$ is introduced as a consequence of the Girsanov transformation, that is needed in cases where $r \neq 0.5 \sigma^{2}$. The same argumentation holds for the other types of Parisians (up/down, out/in), where the minus sign in the exponent disappears for the up-variant of the Parisian contract. Now we use the $\tilde{L}^{*}$ to approximate the value $V_{P D I C}(L)$ by $V_{D I C}\left(\tilde{L}^{*}\right)$. In the next section we show how we deal with Parisians that are already in the excursion.

\section{Valuing Parisians That Are Already in the Excursion}

Here we valuate a Parisian option at time $t>0$. Suppose we are interested in the $P D I C$ with some barrier $L$. In the case $S_{t}<L$, the excursion of the stock path below $L$ has already started and this effects its value. Define $d$ as the time the stock already spent below $L$ by

$$
d:=t-\gamma_{t}
$$

Here we are interested in the case where $d<D$ and another excursion longer than $D$ did not happen yet. It is clear that we have two possibilities now. Either we return to the level $L$ too early to knock in, i.e. we are in the set $\left\{T_{L} \leq D-d\right\}$, or, we are in the complement of this set, and so stay long enough to knock in. Here we abuse the notation $T_{L}$ for the amount of time we have to wait after time $t$ to hit the level $L$, that we should write as $T_{L}^{t}$ given by

$$
T_{L}^{t}=\inf _{s>0}\left\{S_{s+t}=L\right\}
$$

In the same way we will abuse the notation of $T_{D, L}^{-}$. Considering these two cases we could be in, the value of a PDIC is given by

$$
\begin{aligned}
V_{P D I C}(d)= & e^{-r(T-t)} \mathbb{E}\left[\left(S_{T}-X\right)^{+} 1_{\left\{T_{L}>D-d\right\}} \mid \mathcal{F}_{t}\right]+ \\
& e^{-r(T-t)} \mathbb{E}\left[\left(S_{T}-X\right)^{+} 1_{\left\{T_{D, L}^{-}<T-t\right\}} 1_{\left\{T_{L} \leq D-d\right\}} \mid \mathcal{F}_{t}\right]
\end{aligned}
$$

So, in the case that we stay long enough below $L$ we get a standard call, otherwise we get a fresh $P D I C$ for the remaining time to expiry. If we write $1_{\left\{T_{L}>D-d\right\}}=$ $1-1_{\left\{T_{L} \leq D-d\right\}}$, we find for $V_{P D I C}(d)$

$$
V_{P D I C}(d)=V_{B S C}-e^{-r T^{\prime}} \mathbb{E}\left[\left(S_{t} e^{\left(r-\sigma^{2} / 2\right) T^{\prime}+\sigma B_{T^{\prime}}}-X\right)^{+} 1_{\left\{T_{D, L}^{-}>T^{\prime}\right\}} 1_{\left\{T_{L} \leq D-d\right\}}\right] .
$$

Here $V_{B S C}$ is the value of a standard call with time to expiration $T^{\prime}:=T-t$ and we used the Markov property to remove the conditioning. Now we change our measure to $\mathbb{Q}$ in the same way as above. Conditioning on $\mathcal{F}_{T_{L}}$ gives

$$
\begin{aligned}
V_{P D I C}(d)= & V_{B S C}- \\
& e^{-\tilde{r} T^{\prime}} \mathbb{E}_{\mathbb{Q}}\left[1 _ { \{ T _ { L } \leq D - d \} } \mathbb { E } _ { \mathbb { Q } } \left[e^{\left.\left.m Z_{T^{\prime}}\left(S_{t} e^{\sigma Z_{T^{\prime}}}-X\right)^{+} 1_{\left\{T_{D, L}^{-}>T^{\prime}\right\}} \mid \mathcal{F}_{T_{L}}\right]\right],},\right.\right.
\end{aligned}
$$


where $\tilde{r}$ is defined by $\tilde{r}:=r-0.5 m^{2}$. Using the Markov property again, we recognize $V_{P D O C}\left(T^{\prime}-T_{L}\right)$ apart from the proper discounting, so we get

$$
V_{P D I C}(d):=V_{B S C}-e^{m l} \mathbb{E}_{\mathbb{Q}}\left[1_{\left\{T_{L} \leq D-d\right\}} e^{-\tilde{r} T_{L}} V_{P D O C}\left(T^{\prime}-T_{L}\right)\right],
$$

where $l$ is defined by $l=\sigma^{-1} \ln \frac{L}{S_{t}}$. Using the distribution of the hitting time [3] we can calculate the expectation. If we furthermore approximate $V_{P D O C}\left(T^{\prime}-T_{L}\right)$ by $V_{D O C}\left(\tilde{L}^{*}, T^{\prime}-T_{L}\right)$ we obtain

$$
V_{P D I C}(d):=V_{B S C}-\int_{0}^{D-d} V_{D O C}\left(L^{*}, T^{\prime}-u\right) \frac{|l| e^{m l-\tilde{r} u-\frac{l^{2}}{2 u}}}{\sqrt{2 \pi u^{3}}} d u
$$

This integral should be computed numerically. In the example later on we will show that a relatively small number of steps is sufficient to obtain a value close to the true Parisian price. The same way of reasoning could be used to derive prices for the other types of Parisian contracts.

\section{$6 \quad$ Numerical Examples}

As we presented our method as one that can be very useful in practice, we will give some numerical results. Table 1 contains a comparison between implied barrier prices and Laplace prices.

\begin{tabular}{|c|c|c|c|c|c|c|c|}
\hline \multirow{2}{*}{\multicolumn{2}{|c|}{$S_{0}=100, \mathrm{~L}=90$}} & \multicolumn{2}{|c|}{$\mathrm{D}=10 / 365$} & \multicolumn{2}{|c|}{$\mathrm{D}=20 / 365$} & \multicolumn{2}{|c|}{$\mathrm{D}=200 / 365$} \\
\hline & & \multicolumn{2}{|c|}{ Laplace Imp Bar } & \multicolumn{2}{|c|}{ Laplace Imp Bar } & \multicolumn{2}{|c|}{ Laplace Imp Bar } \\
\hline & $\mathrm{X}$ & $\mathrm{T}=1 \mathrm{~T}=2$ & $\mathrm{~T}=1 \quad \mathrm{~T}=2$ & $\mathrm{~T}=1 \mathrm{~T}=2$ & $\mathrm{~T}=1 \mathrm{~T}=2$ & $\mathrm{~T}=1 \mathrm{~T}=2$ & $\mathrm{~T}=1 \quad \mathrm{~T}=2$ \\
\hline & 80 & 6.5411 .36 & $6.55 \quad 11.36$ & $4.97 \quad 9.42$ & $4.99 \quad 9.43$ & 0.261 .68 & $0.35 \quad 1.77$ \\
\hline$r=4.5 \%$ & 90 & $3.84 \quad 8.23$ & $3.84 \quad 8.23$ & $2.79 \quad 6.69$ & $2.80 \quad 6.69$ & $0.08 \quad 0.99$ & 0.14 \\
\hline$\sigma=30 \%$ & 100 & $2.18 \quad 5.92$ & $2.18 \quad 5.92$ & $1.52 \quad 4.72$ & $1.52 \quad 4.72$ & 0.020 .58 & $0.05 \quad 0.64$ \\
\hline & 110 & $1.21 \quad 4.25$ & $1.21 \quad 4.25$ & 0.813 .32 & $0.81 \quad 3.32$ & $0.01 \quad 0.34$ & $0.02 \quad 0.39$ \\
\hline ImpBar & & & 84.5784 .57 & & 82.4282 .42 & & 68.1368 .13 \\
\hline & 80 & 8.5314 .38 & 8.5414 .38 & 6.5111 .98 & 6.5311 .99 & $0.35 \quad 2.21$ & 0.48 \\
\hline $\mathrm{r}=2.5 \%$ & 90 & 5.7411 .30 & $5.75 \quad 11.31$ & $4.24 \quad 9.27$ & 9.28 & $0.15 \quad 1.48$ & 0.24 \\
\hline$\sigma=40 \%$ & 100 & $3.84 \quad 8.91$ & $3.84 \quad 8.91$ & $2.74 \quad 7.20$ & 2.75 & 0.061 .00 & 0.12 \\
\hline & 110 & $2.55 \quad 7.04$ & $2.56 \quad 7.04$ & $1.77 \quad 5.61$ & 1.78 & 0.030 .68 & 0.77 \\
\hline ImpBar & & & 85.4585 .45 & & 80.0480 .04 & & 62.2262 .22 \\
\hline
\end{tabular}

Table 1. Prices for PDIC at $t=0$

Here we see that prices are close to the inverse Laplace prices for $D$ relatively small compared to $T$. Furthermore we see that the implied barrier that is found 
in the upper left corner (i.e. 84.57), is recognized in the implied barrier graph in figure 1 for the same case as shown above. As we would like to use the prices for relatively small $D$, we also need deltas for $D$ relatively large compared to $T$. Table 2 shows that the error in the deltas is much less than that in the prices.

Table 2. Deltas for PDIC at $t=0$

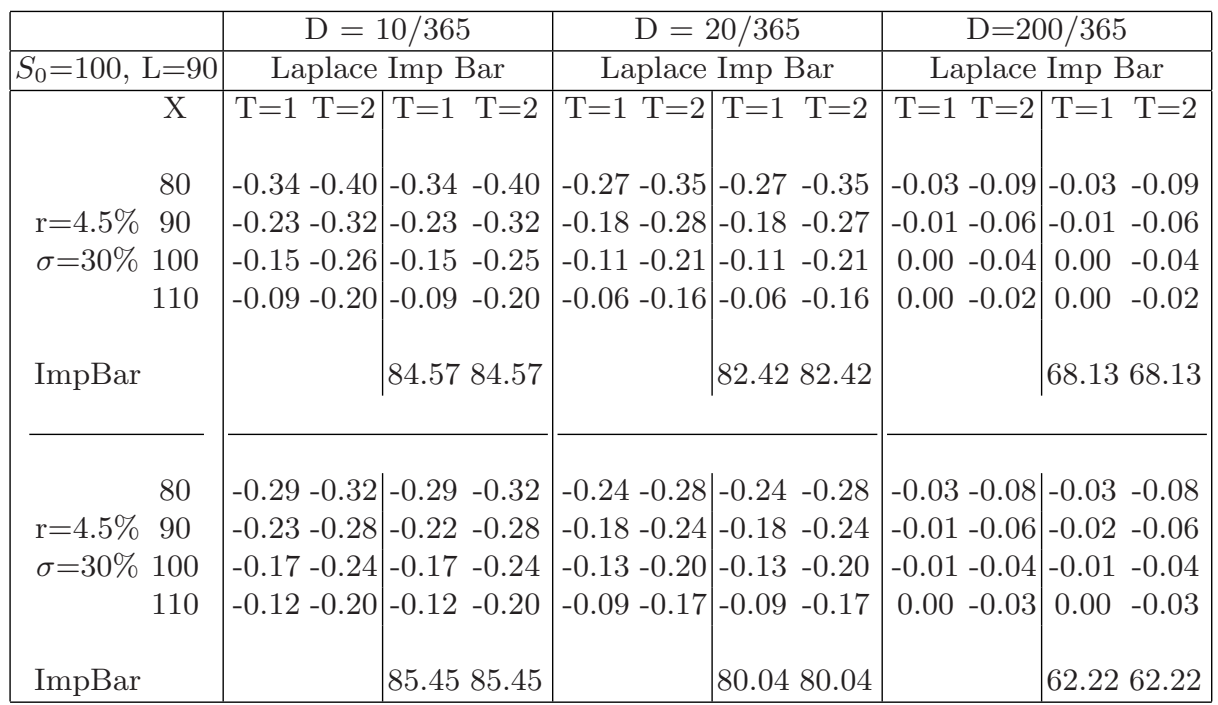

We also computed prices for Parisians based on a stock path that is already in the excursion. Table 3 lists these prices.

Table 3. Price for PDIC at $t>0$ and $d>0$

\begin{tabular}{|c|c|c|c|c|c|c|c|}
\hline \multicolumn{2}{|c|}{$\mathrm{r}=4.5 \%, \sigma=30 \%$} & \multicolumn{2}{|c|}{$\mathrm{D}=10 / 365$} & \multicolumn{2}{|c|}{$\mathrm{D}=20 / 365$} & \multicolumn{2}{|c|}{$\mathrm{D}=200 / 365$} \\
\hline \multicolumn{2}{|c|}{$S_{0}=100, \mathrm{~L}=90$} & \multicolumn{2}{|c|}{ Laplace Imp Bar } & \multicolumn{2}{|c|}{ Laplace Imp Bar } & \multicolumn{2}{|c|}{ Laplace Imp Bar } \\
\hline $\mathrm{X}$ & $\mathrm{d}$ & $\mathrm{T}=1 \quad \mathrm{~T}=2$ & $\mathrm{~T}=1 \quad \mathrm{~T}=2$ & $\mathrm{~T}=1 \quad \mathrm{~T}=2$ & $\mathrm{~T}=1 \quad \mathrm{~T}=2$ & $\mathrm{~T}=1 \mathrm{~T}=2$ & $\mathrm{~T}=1 \quad \mathrm{~T}=2$ \\
\hline 80 & $0.2 \mathrm{D}$ & 13.0418 .56 & 13.0418 .56 & 11.0216 .40 & 11.0216 .41 & 1.404 .16 & 1.51 \\
\hline 100 & $0.2 \mathrm{D}$ & $5.44 \quad 10.77$ & $5.45 \quad 10.77$ & $4.34 \quad 9.26$ & $4.35 \quad 9.26$ & $0.27 \quad 1.75$ & 0.31 \\
\hline 80 & $0.6 \mathrm{D}$ & 13.9819 .52 & 13.9719 .52 & 12.5918 .05 & 12.5918 .05 & $2.79 \quad 5.92$ & 2.95 \\
\hline 100 & $0.6 \mathrm{D}$ & $5.99 \quad 11.46$ & $\begin{array}{ll}5.99 & 11.46\end{array}$ & $\begin{array}{ll}5.22 & 10.43\end{array}$ & $5.22 \quad 10.43$ & $0.82 \quad 2.82$ & 0.86 \\
\hline $\operatorname{ImpBa}$ & & & 84.5784 .57 & & 82.4282 .42 & & 68.1368 .13 \\
\hline
\end{tabular}

As these computations involved numerical integration we have to chose a number of integration steps. In this numerical approximation the interval is divided into 25 steps for $D=10 / 365$ and $D=20 / 365$. We divided it into 250 
steps for $D=200 / 365$. The results are analogous to the former price table. The prices are quite good for $D$ relative small compared to $T$. Again we will compute for this particular case the deltas to show that they do not differ that much, as is shown in table 4 .

Table 4. Deltas for PDIC at $t>0$ and $d>0$

\begin{tabular}{|c|c|c|c|c|c|c|c|c|}
\hline \multicolumn{2}{|c|}{$\mathrm{r}=4.5 \%, \sigma=30 \%$} & \multicolumn{3}{|c|}{$\mathrm{D}=10 / 365$} & \multicolumn{2}{|c|}{$\mathrm{D}=20 / 365$} & \multicolumn{2}{|c|}{$\mathrm{D}=200 / 365$} \\
\hline$S_{0}=100$ & $\mathrm{~L}=90$ & Laplace & $\operatorname{Imp}$ & Bar & Laplace & Imp Bar & Laplace & Imp Bar \\
\hline & $\mathrm{d}$ & $\mathrm{T}=1 \mathrm{~T}=2$ & $\mathrm{~T}=1$ & $\mathrm{~T}=2$ & $\mathrm{~T}=1 \quad \mathrm{~T}=2$ & $\mathrm{~T}=1 \quad \mathrm{~T}=2$ & $\mathrm{~T}=1 \quad \mathrm{~T}=2$ & $\mathrm{~T}=1 \quad \mathrm{~T}=2$ \\
\hline 80 & $0.2 \mathrm{D}$ & $0.03 \quad 0.02$ & 0.02 & 0.02 & $-0.25-0.28$ & $-0.25-0.28$ & $-0.15-0.26$ & $-0.14-0.26$ \\
\hline 100 & $0.2 \mathrm{D}$ & $0.02 \quad 0.02$ & 0.02 & 0.02 & $-0.12-0.19$ & $-0.12-0.19$ & $-0.04-0.13$ & $-0.03-0.13$ \\
\hline 80 & $0.6 \mathrm{D}$ & $\begin{array}{lll}0.28 & 0.29\end{array}$ & 0.28 & 0.29 & $-0.19-0.21$ & $-0.19-0.21$ & $-0.40-0.58$ & $-0.39-0.57$ \\
\hline 100 & $0.6 \mathrm{D}$ & $\begin{array}{ll}0.16 & 0.21\end{array}$ & 0.16 & 0.21 & $-0.09-0.14$ & $-0.09-0.14$ & $-0.14-0.33$ & $-0.13-0.32$ \\
\hline ImpBaI & & & 84.57 & 84.57 & & 82.4282 .42 & & 68.1368 .13 \\
\hline
\end{tabular}

\section{Conclusion}

Valuation of Parisian options is important as popular investment tools like convertible bonds exhibit the Parisian option feature. Well known methods for calculating its price are either computationally slow (PDE method) or inflexible (Laplace method). Here we provide a way to use standard barrier models with an implied barrier for calculating prices. The advantages of these method for trading firms are threefold. First no new model has to be implemented. Moreover it is possible to value the American options with a Parisian constraint, by using the implied barrier in the standard American barrier option. Discrete dividends can be taken into account. Finally a lot has been done pricing barriers in a non-constant volatility environment. Using the implied barrier again yields an approximation of the Parisian price in such an environment.

\section{References}

1. J. Abate, W. Whitt Numerical Inversion of Laplace Transforms of Probability Distributions, ORSA Journal on computing 7(1), 36-43, (1995)

2. M. Chesney, M. Jeanblanc-Pique, M. Yor Brownian Excursions and Parisian Barrier Options, Adv. Appl. Prob. 29, 165-184, (1997)

3. K. L. Chung Excursions in Brownian Motion, Ark. Math. 14, 155-177, (1997)

4. R. T. Durrett, D. L. Iglehart Functionals of Brownian Meander and Brownian Excursions, Ann. Probability 5, 130-135, (1977)

5. A. Grau Moving Windows, master thesis at School of Computer Science, University of Waterloo, Canada (2003)

6. R. J. Haber, P.J. Schönbucher, P. Wilmott An American in Paris, OFRC Working Papers Series 1999-MF-14, 24-29, (1999) 